Ann. Biol. anim. Bioch. Biophys., 1977, 17 (4), 531-534.

\title{
Etude de la différence de potentiel entre la muqueuse iléale et la peau chez l'enfant. Effet d'un régime pauvre en sodium. (Résultats préliminaires)
}

\author{
par C. RICOUR *, P. FEYDY**, P. ARHAN ** \\ * Clinique des Maladies du Rein et du Métabolisme chez l'Enfant \\ ** Département de physiologie, Clinique Chirurgicale Infantile \\ Hôpital des Enfants Malades, 149, rue de Sèvres, 75730 Paris Cedex 15
}

Summary. Electrical potential difference between ileal mucosa and skin in child. Effect of reducing sodium intake.

The potential difference (PD) between ileal mucosa and skin, and urinary excretion of tetrahydroaldosterone (THA) were studied in two children (one with ileostomy and the other with ileocoloplasty) before and during sodium intake reduction. During balanced intake, PD was $24.1 \pm 1 \mathrm{mV}$ and $24.2 \pm 1.8 \mathrm{mV}$; THA was below $40 \mu \mathrm{g} / 24 \mathrm{~h}$. When sodium intake was reduced to $0.3 \mathrm{mEq} / \mathrm{kg} / 24 \mathrm{~h}$, PD increased to $54 \pm 3 \mathrm{mV}$ and $56 \pm 4,3 \mathrm{mV}$, and THA to 409 and $155 \mu \mathrm{g} / 24 \mathrm{~h}$. A positive correlation was shown between DP and THA $(r=0.83, p<0.01)$. The authors believe these data tend to prove that aldosterone plays a part in the small intestine control of sodium conservation. Measurement of ileal PD is proposed with urinary sodium excretion, as a parameter to control sodium balance in children in ileostomy.

Dans un travail antérieur, effectué chez des enfants iléostomisés soumis à une restriction progressive d'apport de sodium, nous avions mis en évidence une conservation de sodium par le grêle ; le rôle de l'aldostérone avait été discuté pour rendre compte de ce phénomène (Ricour, Millot et Balsan, 1973). L'injection d'aldostérone augmentant la réabsorption colique du sodium ef la différence de potentiel entre la muqueuse colique et la peau (Edmonds et Godfrey, 1970), ce travail a pour but d'étudier les variations de la différence de potentiel entre la muqueuse iléale ef la peau, en apport équilibré de sodium et au cours de l'hyperaldostéronisme induit par une restriction sodée.

\section{Matériel et méthodes.}

Deux enfants ayant une aganglionose colique totale ont été étudiés. L'un âgé de 2 ans avait une iléocoloplastie depuis 8 mois, l'autre âgé de 5 ans une iléostomie terminale depuis 18 mois. 
L'étude a été effectuée durant 3 périodes successives de 6 jours. Durant la première ef la troisième périodes les apports de sodium ont été de $15 \mathrm{mEq} / \mathrm{kg} / 24 \mathrm{~h}$ dans un cas, et de $7 \mathrm{mEq} / \mathrm{kg} / 24 \mathrm{~h}$ dans l'autre cas de façon à assurer une natriurèse de $3 \mathrm{mEq} / \mathrm{kg} / 24 \mathrm{~h}$. Au cours de la seconde période, l'apport de sodium a été réduit dans les 2 cas à $0,3 \mathrm{mEq} / \mathrm{kg} / 24$ h sous contrôle de la natrémie et de la courbe pondérale 2 fois par jour. La différence de potentiel a été mesurée tous les deux jours, et l'excrétion urinaire de la tétrahydroaldostérone les deux derniers jours de chaque période.

Mesure de la différence de potentiel.

Cette mesure a été faite à l'aide de deux électrodes en platine réalisées selon les données de Edmonds et Godfrey (1970). L'électrode exploratrice (fig. 1 A) est logée dans une chambre cylindrique d'altuglas de $94 \mathrm{~mm}$ de long ef $10 \mathrm{~mm}$ de diamètre ; à son extrémité se visse un bouchon arrondi comportant un orifice latéral ; le contact entre la muqueuse intestinale ef l'électrode est assuré par une solution de chlorure de sodium (150 mM/l) el d'agar-agar (4 p. 100), introduite dans la chambre contenant l'électrode, immédiatement avant la mesure. L'électrode de référence (fig. 1 B) est située dans un anneau d'altuglas mesurant $20 \mathrm{~mm}$ de diamètre et $15 \mathrm{~mm}$ de haut, le contact étant établi avec la même solution de chlorure de sodium et d'agar-agar. Les électrodes sont reliées à un voltmètre numérique (Schlumberger VM1 240) don† la sensibilité est réglée à $1 / 10$ e de $\mathrm{mV}$.
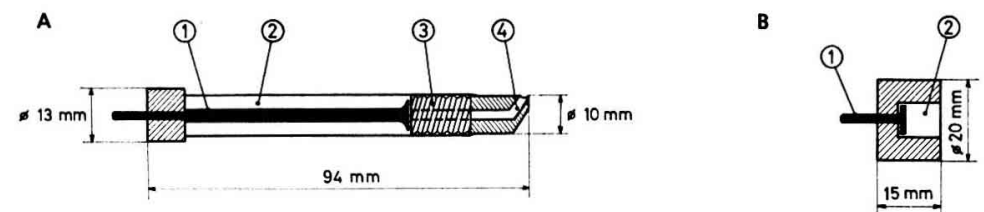

FIG. 1. - Schéma des électrodes utilisées. A : Electrode exploratrice. B : Electrode cutanée.

(1) électrode ; (2) chambre en polyester ; (3) bouchon obturateur ; (4) orifice latéral.

L'électrode de référence cutanée est appliquée sur le quadrant supéro-externe d'une fesse. L'électrode exploratrice est introduite dans la lumière intestinale, dont on a préalablement vérifié la vacuité, de 3 à $5 \mathrm{~cm}$ de la marge cutanée; elle est ensuite inclinée latéralement afin d'établir un contact correct entre son extrémité et la muqueuse. Dix secondes sont nécessaires pour obtenir la stabilisation des valeurs indiquées sur le voltmètre. La constitution des électrodes pouvant être à l'origine d'une différence de potentiel, on soustrait de la première valeur mesurée, la valeur obtenue en appliquant les deux électrodes sur la peau. On obtient ainsi la différence de potentiel qui est due à la paroi intestinale.

Dosage de la tétrahydroaldostérone.

La mesure de la téłrahydroaldostérone dans les urines a été effectuée selon la technique de Legrand et al. (1967) ; son excrétion moyenne par jour est exprimée en $\mu \mathrm{g} / 24 \mathrm{~h}$. 
Résultats (fig. 2).

La différence de potentiel entre la muqueuse iléale et la peau au cours des deux périodes d'équilibre hydroélectrolytique est de $24,1 \pm 1 \mathrm{mV}(\mathrm{n}=6)$ dans un cas et de $24,2 \pm 1,8(n=5)$ dans l'autre cas ; durant la restriction d'apport de sodium les

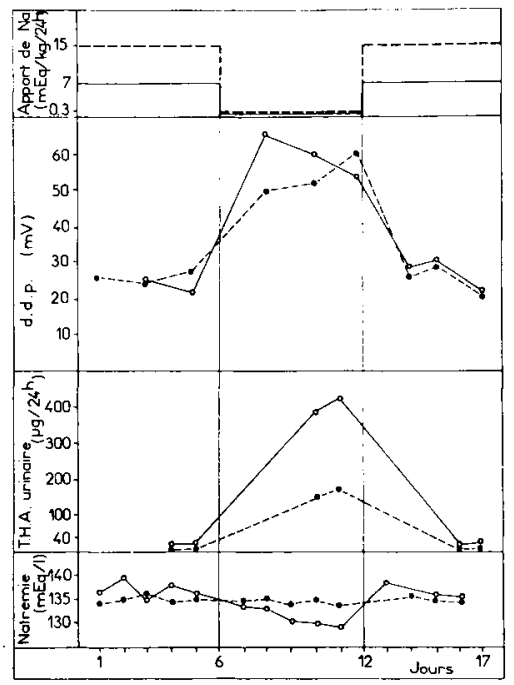

FIG. 2. - Représentation schématique des variations de la différence de potentiel iléale (d. d. p.), de l'excrétion urinaire de la tétrahydroaldosférone (THA) el de la natrémie en fonction de l'apport de sodium.

Etude réalisée chez 2 enfants l'un en iléostomie (traits pointillés), l'autre en iléocoloplastie (traits pleins).

valeurs s'élèvent respectivement à $54 \pm 3 \mathrm{mV}(\mathrm{n}=3)$ et $56 \pm 4,3 \mathrm{mV}(\mathrm{n}=3)$, ces variations de la différence de potentiel entre les phases d'équilibre ef de restriction sodées étant très significatives $(P<0,001)$. L'excrétion urinaire de la tétrahydroaldostérone est, dans les deux cas, inférieure à $40 \mu \mathrm{g} / 24 \mathrm{~h}$ en période d'équilibre ; elle s'élève respectivement à $409 \mu \mathrm{g} / 24 \mathrm{~h}$ et $155 \mu \mathrm{g} / 24 \mathrm{~h}$. Une corrélation positive est ainsi mise en évidence entre la différence de potentiel iléale et l'excrétion de la tétrahydroaldostérone urinaire $(r=0,83, p<0,01, n=12)$. Il est à noter que durant la restriction d'apport de sodium, la natrémie est demeurée normale chez l'enfant ayant une iléocoloplastie, par contre elle s'est abaissée à $129 \mathrm{mEq} / \mathrm{l}$ chezl'enfanf iléostomisé.

\section{Discussion.}

Ces résultats montrent qu'il est possible de mesurer la différence de potentiel iléale chez l'enfant par une technique simple et non agressive. Cette différence de potentiel déterminée entre la face muqueuse de la paroi iléale et la peau peut, en fait, être assimilée à la différence de potentiel transmurale de l'iléon ; il a été en effet démontré qu'il n'y a aucune différence de potentiel entre une électrode cutanée 
et une électode intraveineuse (Archampong et Edmonds, 1972), ni entre cette dernière et une électrode intrapéritonéale (Turnberg et al., 1970).

Au cours des périodes d'équilibre hydroélectrolytique, sans hyperaldostéronisme, les valeurs que nous avons trouvées, $24,1 \pm 1$ et 24,2 $\pm 1,8 \mathrm{mV}$, sont très voisines, quoique plus élevées, de celles qui ont été rapportées chez des adultes iléostomisés

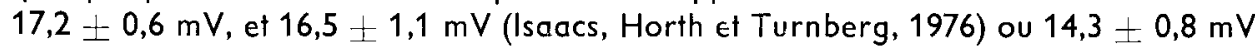
(Prado et al., 1974), lorsque la mesure est effectuée comme dans nos cas à moins de $5 \mathrm{~cm}$ de la marge culanée.

Au cours de l'hyperaldostéronisme induit par la restriction d'apport de sodium à $0,3 \mathrm{mEq} / \mathrm{kg} / 24 \mathrm{~h}$, nous avons mis en évidence une chute de la concentration moyenne du sodium iléal à $59 \mathrm{mEq} / \mathrm{l}$ et une élévation de celle du potassium à $55 \mathrm{mEq} / 1$; une corrélation très significative a été ainsi démontrée entre ces variations d'électrolytes intraluminaires et l'excrétion urinaire de la tétrahydroaldostérone (Ricour, Millot ef Balsan, 1973). L'élévation de la différence de potenticl iléal que nous avcns observée dans les mêmes conditions d'hyperaldostéronisme, et sa corrélction positîive avec la tétrahydroaldostérone urinaire, nous semblent constituer un argument supplémentaire en faveur du rôle joué par l'aldostérone dans le contrôle de la conservation du sodium par le grêle. La réduction de la différence de potentiel iléal constatée par Isaacs, Horth et Turnberg (1976) sous spironolactone, antagoniste de l'aldostérone, renforce cette hypothèse.

En pratique en raison de la simplicité et la rapidité de la mesure de la différence de potentiel iléocutanée ou rectoculanée chez l'enfant, cet examen nous paraît être, au même titre que la natriurèse (Ricour, Millot et Balsan, 1973), un paramètre de surveillance important pour l'équilibration des syndromes de perte de sodium d'origine digestive chez l'enfant.

Commission CNERNA Digestion-Absorption, Tours, 13 novembre 1976,

Remerciements. - Nous remercions le professeur J. C. Legrand qui a effectué les dosages urinaires de la tétrahydroaldostérone.

\section{Références}

ARCHAMPONG E. Q., EDMONDS C. J., 1972. Effect of luminal ions on the transepithelial electrical potential difference of human rectum. Gut 13, 559-565.

EDMONDS C. J., GODFREY R. C., 1970 . Measurement of electrical potentials of the human rectum and pelvic colon in normal and aldosterone treated patients. Gut, 11, 330-337.

ISAACS P. E. T., HORTH C. E., TURNBERG L. A., 1976. The electrical potential difference across human ileostomy mucosa. Gastroenterology, 70, 52-58.

LEGRAND J. C., LEGRAND S., ZOGBI F., GUILLEMANT S., 1967. Détermination quantitative de la tétrahydroaldostérone. Ann. Biol. Clin., 25, 1199-1209.

PRADO P (de), MORAES-FILHO J., SALAS-COLL C., BLENDIS L. EDMONDS C. J., $1974 . \quad$ Electrical potential difference and absorption of water, sodium, and potassium by the terminal ileum of ileostomy patients. Gut, 15, 977-981.

RICOUR C., MILLOT M., BALSAN S., 1973. Sodium conservation after total or subtotal colonic resection in children. Scand. J. Gastroent., 8, 743-750.

TURNBERG L. A., BIEBERDORF F. A., MORAWSKI S. G., FORDTRAN J. S., 1970. Interrelationships of chloride, bicarbonate, sodium and hydrogen transport in the human ileum.. J. Clin. Invesi., 49, 557-567. 